\title{
Althusser y Wittgenstein: ideología y análisis terapéutico del lenguaje
}

\author{
Althusser and Wittgenstein: \\ ideology and therapeutic analysis of language
}

Pedro Diego Karczmarczyk ${ }^{1}$

\section{RESUMEN}

En el presente trabajo pretendemos establecer las bases para realizar una comparación poco transitada, la de la perspectiva de Althusser sobre la ideología y el abordaje del lenguaje que surge en la perspectiva del segundo Wittgenstein. Intentaremos mostrar la existencia de un paralelo entre las consideraciones de Althusser sobre la constitución de los individuos en sujetos a través del funcionamiento de "la categoría de sujeto" y un movimiento análogo implicado en las observaciones de Wittgenstein acerca de la constitución del hablante en los juegos de lenguaje, que si nuestra interpretación es válida, debería describirse como "interpelación de los individuos a través de la categoría de significado".

Palabras clave: Ideología. Subjetividad. Práctica. Juego de lenguaje. Significado.

\begin{abstract}
In this paper we intend to establish the basis for conducting a comparison not usually made between Althusser's perspective concerning ideology and Wittgenstein's one in relation to his language approach. We try to show a parallel between the remarks of Althusser on the constitution of individuals in subjects "through the category of subject" and an analogous movement implied in Wittgenstein own remarks on the constitutions of speakers in language-games, which, if our interpretation is right, should be described as the "interpellation of individuals through the category of meaning".
\end{abstract}

Keywords: Ideology. Subjectivity. Practice. Language-game. Meaning.

\footnotetext{
${ }^{1}$ Universidad Nacional de La Plata (UNLP) /Consejo nacional de Investigaciones científicas y técnicas (CONICET) - Argentina. peterkado@yahoo.com
}

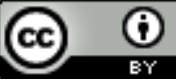

Esta obra foi licenciada com uma Licença Creative Commons - Atribuição 3.0 Não Adaptada. 
En el presente trabajo pretendemos establecer las bases para realizar una comparación poco transitada, la de la perspectiva de Althusser sobre la ideología y el abordaje del lenguaje que surge en la perspectiva del segundo Wittgenstein. Intentaremos mostrar la existencia de una importante semejanza entre las consideraciones de Althusser sobre la constitución de los individuos en sujetos a través del funcionamiento de "la categoría de sujeto" y un movimiento análogo implicado en las observaciones de Wittgenstein acerca de la constitución del hablante. $^{2}$

Esta estrategia analítica se justifica por los aspectos que la misma ilumina en ambas direcciones, dejando ver puntos débiles y desarrollar mejor otros, y porque permite percibir consecuencias inesperadas en ambas posturas. Además, la lectura propuesta fortalece de algún modo ambas posiciones. Del lado de Althusser, porque se beneficia de algunos de los argumentos más detallados que pueden encontrase en la filosofía contemporánea para establecer algunos de sus puntos básicos (como el del carácter externo y social de las prácticas simbólicas). Por el lado de Wittgenstein, porque leerlo a través de Althusser implica un aporte estimable al debate sobre las consecuencias políticas de su filosofía, posibilitando otros cruces y enriquecimientos. Por último, las divergencias entre ambos permiten una mejor comprensión de las tensiones a las que se ve sometida la reflexión filosófica en ambos autores.

Nuestro argumento remite a una analogía entre las líneas centrales de ambos proyectos, la que probablemente se deriva de la semejanza de los blancos a los que dirigen sus ataques. Althusser lleva adelante un rechazo radical de una concepción de la ideología que podría ser calificada de "idealista" y que puede encontrarse en varias reflexiones marxistas, a pesar de su profesión de fe materialista, apoyándose en ciertos aspectos de la presentación de esta noción en La ideología alemana y otros textos clásicos. Este movimiento puede alinearse con la crítica que Wittgenstein dirige a lo que denominamos "concepción mentalista clásica" en semántica y en epistemología. Por ésta entendemos la concepción comprometida con la existencia de un dominio (tradicionalmente ideas, aunque su rol también puede ser otorgado a otros estados de un individuo) que tiene simultáneamente

\footnotetext{
${ }^{2}$ Nuestro análisis se concentrará básicamente en ALTHUSSER 1970 y en WITTGENSTEIN 1953, obra que citamos según el número de parágrafo para la primera parte y según la página para la segunda parte.

Cad. de Pesq. Interdisc. em Ci-s. Hum-s., Florianópolis, v.12, n.101, p.4-30 ago/dez 2011
} 
relevancia normativa y explicativa en relación a nuestra conducta, lingüística y no lingüística. Este dominio desempeñaría el papel de una fuente de la que nuestro comportamiento emana (rol explicativo) a un tiempo que debe ser concebido como inmediatemente accesible a la conciencia, como una fuente que provee razones para actuar en circunstancias particulares (rol normativo).

\section{LA REFORMULACIÓN DE LA PROBLEMÁTICA DE LA IDEOLOGÍA POR}

\section{ALTHUSSER}

Althusser cuestiona la visión clásica de la ideología, a la que él mismo caracteriza en estos términos: "...en la ideología "los hombres se representan en forma imaginaria sus condiciones reales de existencia”" (1970 p. 44) La ideología sería así un mecanismo doble, de ilusión/alusión. En consecuencia, una vez en posesión de la clave de la deformación (ilusión) operada en las representaciones ideológicas, por medio de un proceso de interpretación, podríamos acceder a las condiciones reales de existencia que se expresan (alusión) en la ideología de manera deformada.

De acuerdo a esta concepción, la pregunta crucial de la crítica ideológica inquiere por el origen de las representaciones ideológicas, es decir, se pregunta por la identificación del factor, la causa, que las distorsiona, por oposición a lo que podríamos entender como una representación adecuada de la realidad social. Las respuestas que esta pregunta ha recibido a lo largo de la historia son variadas: desde la teoría ilustrada del "engaño del clero" hasta la teoría hegelianofeuerbachiana suscripta por el joven Marx, donde la representación alienada (imaginaria) de las condiciones reales de existencia se debe a la existencia de una contradicción en las condiciones reales, debido a que la reproducción de la existencia de la humanidad se da a través de la negación de su esencia (trabajo alienado, alienación). (ALTHUSSER, 1970, pp. 44-45) 
Althusser propone una concepción alternativa de la ideología, colocando esta noción en el marco de otra "problemática" ${ }^{3}$ La nueva concepción es presentada a través de una serie de tesis. La primera de ellas indica: "La ideología representa la relación imaginaria de los individuos con sus condiciones reales de existencia" (1970, p. 43). Esta nueva caracterización realiza un movimiento radical en el campo del saber, reemplazando de las preguntas que deben ser planteadas:

\begin{abstract}
si esto es así [si lo que la ideología 'representa' es la relación imaginaria de los individuos con sus condiciones de existencia, y no las condiciones reales de la producción de manera distorsionada] la pregunta por la causa de la deformación imaginaria de las condiciones de existencia en la ideología desaparece y debe ser reemplazada por otra: "¿por qué la representación dada a los individuos de su relación (individual) con las relaciones sociales que gobiernan su existencia y su vida colectiva e individual es necesariamente imaginaria? ¿Y cual es la naturaleza de este ente imaginario? ["quelle est la nature de cet imaginaire?']"(1970, p. 46) ${ }^{4}$
\end{abstract}

Detengámonos en la formulación althusseriana, para poder apreciar con mayor claridad las consecuencias de esta sustitución de preguntas. Un poco más adelante se sostiene:

Lo representado en la ideología es ante todo la relación que existe entre ellos y sus condiciones de existencia. Tal relación es el punto central de toda representación ideológica, y por lo tanto imaginaria, del mundo real. En esta relación está contenida la causa que debe dar cuenta de la deformación imaginaria de la representación ideológica del mundo real. 0 más bien, para dejar en suspenso el lenguaje causal, es necesario emitir la tesis de que es la naturaleza imaginaria de esa relación [la del individuo con sus condiciones reales] la que sostiene toda la deformación imaginaria que se puede observar (si no se vive en su verdad) en toda ideología. (1970, p. 45-46).

Es decir, la ideología no sería tanto un cuadro surrealista, una variación imaginaria (deformada) de la realidad, sino, como lo propone en algunas de sus viñetas Miguel Rep, una representación fidedigna de una realidad que es en si misma "surrealista". ${ }^{5}$ Es útil retomar aquí la noción de una "representación ideológica

\footnotetext{
${ }^{3}$ Para Althusser la problemática es el conjunto de conceptos y sus combinaciones que constituye el suelo y el horizonte dentro del que cualquier discurso (científico, filosófico, ideológico) encuentra sus problemas. Dos nociones clave en el planteo althusseriano dependen de esta noción, la de lectura sintomal (que establece el vínculo entre un conjunto de enunciados y su problemática) y la de ruptura epistemológica (que da cuenta de los movimientos discursivos que producen un cambio de problemática). Véase KARSZ, 1970: cap. 1, § 3, pp. 58 y ss.

4 Aquí, como en el resto del trabajo, indicamos nuestros añadidos encerrándolo entre corchetes cuadrados "[]". Al comienzo del Cuaderno azul Wittgenstein apela un mecanismo análogo de reemplazo de preguntas. Véase WITTGENSTEIN, 1958, p. 27.

5 Rep suele producir este efecto, representando en sus viñetas al pintor, su tela -un cuadro famoso reputado como no representativo- y el modelo a ser representado, generando un efecto sorprendente, al sugerir que el prodigio no estaría en el cuadro, sino en la realidad que el cuadro representa. Véase la serie "Bellas artes" en www.miguelrep.com.ar [acceso en 2/6/2011].
}

Cad. de Pesq. Interdisc. em Ci-s. Hum-s., Florianópolis, v.12, n.101, p.4-30 ago/dez 2011 
de la ideología" o la análoga "ideología de la ideología" (ALTHUSSER, 1970, p. 48). La concepción ideológica de la ideología contrasta, como veremos, con la "ideología en estado práctico". La representación ideológica de la ideología, la que habla, por ejemplo, en sintonía con La ideología alemana, de los valores e ideas como principios de acción, no es una representación distorsionada, sino una representación fidedigna de una distorsión que ocurre en otra parte, en la ideología en estado práctico. La deformación no está entonces en el proceso de representación, sino en lo que es representado en la ideología, la propia relación de los individuos con sus condiciones de existencia.

El cambio de preguntas desbloquea una cuestión que estaba obturada por una concepción de la relación entre la infraestructura económica y la superestructura jurídico-política modelada en términos de una "metáfora espacial". Se trata de la concepción de esta relación en los términos de un edificio en el que la base (estructura económica o infraestructura) sostiene a los pisos construidos sobre ella (la superestructura). Althusser le reconoce un valor "pedagógico" a esta metáfora, debido a que señala cómo la base "determina en última instancia" lo que sobre ella se apoya. Althusser había sostenido poco antes en este mismo texto que sólo la perspectiva de la reproducción, comprendida en un sentido amplio, como la reproducción de las fuerzas productivas (medios de producción y fuerza de trabajo) y de las relaciones de producción, una perspectiva por definición dinámica, permitiría desarrollar una teoría adecuada de la relación entre base y superestructura. Al cuestionar qué es causa y qué efecto en esta relación, aunque sin decirlo explícitamente, la metáfora tópica, estática, es identificada como un "obstáculo epistemológico" (BALIBAR, 2004a). Reconsiderar la cuestión no implicará buscar nuevas (mejores) respuestas para las preguntas que surgen en el seno de la metáfora espacial, sino, por el contrario, la disolución de sus problemas y su reemplazo por otros. No se trata entonces tanto de una nueva teoría de la relación estructura-superestructura como de un cambio de la noción de estructura en cuanto tal. 6

\footnotetext{
${ }^{6}$ Balibar caracteriza de la siguiente manera el contexto en el que se produjeron las obras de Althusser: "A la obra del propio Marx se debería la explicación de las leyes económicas del capitalismo, a la que ahora convendría agregar la explicación de la superestructura. Y lo que es más, desde el punto de vista filosófico la clave de esa adición era invariablemente buscada en la dialéctica del ser (social) y la conciencia (social) (...) Engels, pero también Kautsky, Plejanov, Lenin, Labriola, Gramsci, e incluso Lukács o Sartre, a pesar de todas sus divergencias, se alinearon en torno a esos Cad. de Pesq. Interdisc. em Ci-s. Hum-s., Florianópolis, v.12, n.101, p.4-30 ago/dez 2011
} 
La cuestión que dominaba la metáfora espacial, acerca de la relación entre la estructura, entendida como concreta y real, con la superestructura, comprendida como más o menos irreal, epifenoménica, o superveniente de aquélla, se disuelve, para dar lugar al estudio de la ideología en términos de su materialidad. El nuevo marco evita comprender la reproducción de la fuerza de trabajo como un proceso que fuera en un primer momento el de su reproducción material (la reproducción biológica de los individuos, p. e.) y luego su amoldamiento a las necesidades del sistema social. En su lugar Althusser nos propone pensar en un proceso en el que ambos aspectos ocurren de manera inescindible: "la reproducción de su calificación [de la fuerza de trabajo] (...) [acaece] al mismo tiempo que la reproducción de su sumisión a las reglas del orden establecido." (1970, p. 14)

Este enfoque nos sitúa en un nivel de análisis que si bien es el de la estructura de la sociedad de clases, de las sociedades históricas, permite extraer conclusiones de carácter omnihistórico. El análisis va a mostrar que el mecanismo general de la ideología, o la ideología en general, no posee un carácter histórico, es decir, una caducidad temporal posible. Así, el discurso sobre la ideología en general designa un mecanismo omnihistórico, un mecanismo presente en cualquier formación social, en la medida en que el mismo es una condición de posibilidad de éstas. Mientras la ideología (en cuanto mecanismo de subjetivación) es históricamente invariante, las ideologías particulares son, en cambio, históricamente variables.

La segunda tesis nos llevará a una mejor comprensión de la pregunta inaugurada por su nueva caracterización de la ideología. En esta tesis, Althusser responde a la pregunta presupuesta por este planteo (¿cuál es la naturaleza de ese ente imaginario [a través del cual los individuos se relacionan con, es decir, viven, sus relaciones sociales y que se representa en la ideología]?). En el desarrollo de la misma encontraremos los elementos para responder a la primera (¿Por qué esto ocurre necesariamente?). La segunda tesis enuncia: "La ideología tiene una existencia material" (1970, p. 47).

postulados (...). La posición de Althusser, fue exactamente la inversa. (...) No se propone agregar una teoría de la "superestructura" a la teoría existente de la "estructura", sino por el contrario, transformar el propio concepto de estructura, mostrando que "producción" y "reproducción" son procesos que dependen originariamente de condiciones ideológicas inconscientes." BALIBAR 2004b, pp. 86-87.

Cad. de Pesq. Interdisc. em Ci-s. Hum-s., Florianópolis, v.12, n.101, p.4-30 ago/dez 2011 
El primer paso para explicar esta tesis consiste en caracterizar la forma que toma "la relación imaginaria de los individuos con sus condiciones reales", es decir, lo que se representa en la ideología. Althusser caracteriza así a la "relación imaginaria":

Un individuo cree en Dios, o en el Deber o en la Justicia. Tal creencia depende (para todo el mundo, o sea, para los que viven en una representación ideológica de la ideología, que reduce la ideología a ideas dotadas por definición de existencia espiritual) de las ideas de dicho individuo, por lo tanto, de él mismo en tanto sujeto poseedor de conciencia en la cual están contenidas las ideas de su creencia. (1970, p. 48)7

Colocándonos en el interior del mecanismo ideológico así descrito, se supone que el comportamiento material del sujeto se deriva del mismo naturalmente (ver 1970 , p. 48). Otra formulación del mismo punto introduce un matiz adicional, cuya importancia tendremos ocasión de apreciar más adelante:

\begin{abstract}
...la representación ideológica de la ideología está obligada a reconocer que todo "sujeto" dotado de una "conciencia" y que cree en las "ideas" que su "conciencia" le inspira y acepta libremente, debe por tanto traducir en los actos de su práctica material sus propias ideas de sujeto libre. Si no lo hace, eso "no está bien". (1970, p. 49)
\end{abstract}

El segundo paso consiste en mostrar que esta "representación ideológica de la ideología" posee existencia material. Para ello, Althusser toma nota del reconocimiento, interno a la propia concepción ideológica de la ideología, de que las ideas se traducen en actos. ${ }^{8}$ A partir de esta indicación y siguiendo la pauta para una subversión del orden la representación ideológica de la ideología contenida en el pensamiento de Pascal: "Arrodillaos, moved los labios en oración y creeréis" (cit. en ALTHUSSER, 1970, p. 50), Althusser sostiene que las ideas sólo existen por medio de "actos insertos en prácticas, (...) reguladas por rituales en los cuales se inscriben, en el seno de la existencia material de un aparato ideológico" (1970 p. 49).

Vemos así que la relación imaginaria de los individuos con sus condiciones reales consiste, para los individuos devenidos sujetos y para la representación

7 El concepto de una relación imaginaria es un concepto que Althusser toma de Lacan. En un renombrado texto, Lacan sostiene: “...el estadio del espejo es un drama cuyo empuje interno se precipita de la insuficiencia a la anticipación; y que para el sujeto, presa de la ilusión de la identificación espacial, maquina las fantasías que se sucederán desde una imagen fragmentada del cuerpo hasta una forma que llamaremos ortopédica de sus totalidad -y a la armadura por fin asumida de una identidad enajenante-, que va a marcar con su estructura todo su desarrollo mental." (LACAN, 2005, p. 110).

8 "En todos los casos la ideología de la ideología reconoce, a pesar de su deformación imaginaria, que las "ideas" de un sujeto humano existen o deben existir en sus actos, y si eso no sucede, le proporciona otras ideas correspondientes a los actos (aún perversos) que el sujeto realiza." (ALTHUSSER, 1970 p. 49).

Cad. de Pesq. Interdisc. em Ci-s. Hum-s., Florianópolis, v.12, n.101, p.4-30 ago/dez 2011 
ideológica de la ideología en la que se refleja esta relación, en la transformación de la relación básica o real (práctica y material), en una relación idealista y representacionalista.

La ruptura implicada por la nueva pregunta sobre la ideología (¿por qué los individuos tienen necesariamente una representación imaginaria de la relación con sus condiciones reales?) tiene una expresión paradigmática en la "desaparición" del término "ideas" ("en tanto que dotadas de una existencia ideal, espiritual" aclara Althusser), es decir, justamente aquello que en la concepción tradicional de la ideología a la vez explicaba (los actos de los individuos-sujetos) y era aquello que debía explicarse (en tanto que representaciones deformadas). Aunque Althusser insiste, por su parte, en que en el nuevo esquema subsisten algunos de los viejos términos: "sujeto", “conciencia”, “creencia”, "actos", hay que notar que los mismos han sido radicalmente transformados, por ejemplo "acto" ya no será el comportamiento que brota naturalmente, o de manera adecuada, de una idea, sino el comportamiento que cumple (o no cumple) con los criterios inmanentes a una práctica.

La operación althusseriana nos lleva a abandonar la primacía (ontológica y explicativa) de la concepción de un sujeto que actúa determinado por ideas, para reemplazarla por la concepción de un sujeto que "es actuado" de acuerdo a un sistema que "enunciado en su orden de determinación real" se presentaría del siguiente modo: "ideología existente en un aparato ideológico material que prescribe prácticas materiales reguladas por un ritual material, prácticas éstas que existen en los actos materiales de un sujeto que actúa con toda conciencia según su creencia." (1970, p. 51).

La clave de esta formulación es la noción de sujeto, que será el núcleo de la tercera tesis. Para ello, Althusser propone dos tesis conjuntas: “a) No hay práctica sino por y bajo una ideología; b) No hay ideología sino por el sujeto y para los sujetos." (1970, p. 51), las que confluyen en la tercera y decisiva tesis: "La ideología interpela a los individuos como sujetos." (1970, p. 52).

Al explicar la articulación entre estas tesis Althusser puntualiza que "sólo existe ideología para los sujetos concretos, y esta destinación de la ideología es posible solamente por el sujeto, es decir, por la categoría de sujeto y su funcionamiento" (1970, p. 52) 
Dijimos antes que el trastocamiento operado por las preguntas que nos ocupan nos lleva a reemplazar la concepción de un sujeto fundante por la concepción de un sujeto que "es actuado" de acuerdo a un sistema. Tal sistema no es otro que la propia ideología, dentro de la cual el ritual central es el de la categoría de sujeto, que se materializa en lo que Althusser llama interpelación. La interpelación, podemos decir entonces, es el ritual en el que consiste el funcionamiento de la categoría de sujeto. ${ }^{9}$

La elucidación del funcionamiento de la categoría de sujeto en términos del ritual de la interpelación implica un cuestionamiento radical del lugar otorgado a la categoría de sujeto, como suelo fundante o primer escalón seguro en la filosofía, en la medida en que esta categoría cambia su carácter básico por uno derivado: los individuos llegan a ser tales (llegan a ser sujetos) sólo cumpliendo los requisitos que sobre los mismos impone el ritual de la interpelación (el funcionamiento de la categoría de sujeto).

Sin embargo, el punto señalado por Althusser es un poco más difícil de asir, ya que el mismo alude a un proceso de "doble constitución" en el cual los individuos son convertidos en sujetos a través de su adecuación a los requisitos que impone el funcionamiento de la categoría de sujeto, pero el ritual de la interpelación, el funcionamiento de la categoría de sujeto, es interno a la ideología, a la perspectiva de los sujetos, esto es, de los sujetos concretos: “...la categoría de sujeto es constitutiva de toda ideología sólo en tanto toda ideología tiene por función (función que la define) la constitución de los individuos concretos en sujetos." (1970, p. 52).

La ideología (en particular cuando consideramos la categoría de sujeto) estaría entonces sometida a una paradoja doble. Por una parte, la ideología (incluida la categoría de sujeto, y su funcionamiento, los rituales que la mantienen en la existencia) sólo existe por y a través de los sujetos concretos. Parecería entonces que de la ideología así entendida se pueden pensar un inicio en la misma medida en que se puede pensar un inicio del lenguaje. La noción althusseriana sirve para desplazar la idea de un sujeto fundante, propia de la representación ideológica de la ideología, pero en sus propios términos nos plantea constantemente el problema del huevo y la gallina: los sujetos sólo existen en virtud de la categoría de sujeto, al

\footnotetext{
9 En términos wittgensteinianos, se diría que la interpelación es un elemento constitutivo de la gramática de 'sujeto'. Para la noción de elemento constitutivo, véase WITTGENTEIN, 1953 § 136.

Cad. de Pesq. Interdisc. em Ci-s. Hum-s., Florianópolis, v.12, n.101, p.4-30 ago/dez 2011
} 
insertarse los individuos en los rituales que hacen efectivo el funcionamiento de esta categoría, cuyo núcleo es la interpelación, pero la categoría misma, categoría ideológica por excelencia, sólo existe a través de los sujetos concretos ("sólo existe ideología para los sujetos concretos" (1970, p. 52)), es decir, pertenece a la "representación ideológica de la ideología".

Por otra parte, si atendemos más detalladamente al mecanismo de la interpelación, que se ejemplifica con situaciones concretas de llamados a individuos en los cuales estos reconocen que es a ellos justamente a quienes va dirigido el llamado (“iEh usted, oiga!” por ejemplo) encontramos otra paradoja:

...los individuos son siempre ya interpelados por la ideología como sujetos,
lo cual necesariamente nos lleva a una última proposición: los individuos
son siempre ya sujetos. Por lo tanto los individuos son abstractos respecto
de los sujetos que ellos son siempre ya. Esta proposición puede parecer
una paradoja. (1970, p. 57 )

Volveremos sobre esta cuestión al ocuparnos de Wittgenstein. Pero nuestro recorrido nos permite ya plantearnos y responder el primer interrogante abierto por la nueva caracterización de la ideología por Althusser: “¿por qué la representación dada a los individuos de su relación (individual) con las relaciones sociales que gobiernan su existencia y su vida colectiva e individual es necesariamente imaginaria?" (1970, p. 46).

Nuestro análisis del texto de Althusser ha puesto de manifiesto que la relación de los individuos con sus condiciones de existencia es imaginaria porque los individuos se relacionan con sus condiciones de existencia inscribiéndose en los rituales a través de los cuales funciona la categoría de sujeto. Esto es, los individuos experimentan sus condiciones de existencia como sujetos y ésa es, para ellos, la relación primaria. Cuando Althusser sostiene que "los individuos son siempre ya sujetos" y que, por lo tanto, "los individuos son abstractos en relación a los sujetos que ellos mismos son siempre ya" (1970, p. 57) nos está indicando que los individuos concretos, esa realidad material podríamos decir, está en el orden de la experiencia del individuo-subjetivado después que el "sujeto", es decir, el estatus social está en el orden de la experiencia antes que la realidad material que la soporta. $^{10}$ De una manera semejante, tenemos que repetir insistentemente una

\footnotetext{
${ }^{10}$ Vale la pena aquí recordar la importancia que Althusser da a la tarea de desarrollar una teoría de las modalidades de la materialidad (ALTHUSSER, 1970, p. 48). Por lo tanto, cuando decimos, "la realidad material que la soporta" no nos referimos ni primera ni únicamente a procesos cerebrales, conexiones neuronales, etcétera.

Cad. de Pesq. Interdisc. em Ci-s. Hum-s., Florianópolis, v.12, n.101, p.4-30 ago/dez 2011
} 
palabra para que la misma suene para nosotros despojada de significado, como un mero sonido. (WITTGENSTEIN, 1953, p. 491)

Pero estas observaciones permanecen en un nivel de análisis que podríamos llamar fenomenológico. La clave de la respuesta requiere que prestemos atención a los requisitos que los individuos deben satisfacer para ser clasificados o "reclutados" como sujetos. Para comenzar a entremezclar las reflexiones de Althusser con las de Wittgenstein, podríamos decir que, dentro del lenguaje, todo funciona como si la palabra recibiera su significado en virtud de la intención que asocio a la misma. Para hacer ahora una afirmación que luego tendremos que matizar: así como el desconocimiento de los mecanismos y prácticas que constituyen el funcionamiento de la categoría de significado, donde la seguridad práctica y la ausencia de duda forman parte de los criterios que hacen de alguien un hablante, el desconocimiento de los mecanismos a través de los cuales funciona la categoría de sujeto, donde la seguridad práctica, la ausencia de duda al expresar las propias ideas y pensamientos, etc., comportarse como sujeto en suma, es uno de los requisitos que los individuos han de cumplir para ser sujetos (es decir, para ser incluidos en el funcionamiento de la categoría de sujeto). El funcionamiento de la categoría de sujeto, el trabajo del ritual de la interpelación, queda así oculto en la propia evidencia que el mismo reclama a los individuos para ser reclutados como sujetos. La descripción del mecanismo de la interpelación, en la cual el énfasis está puesto en el hecho de que los individuos responden a la misma sin dudar, ilustra bien el punto que estamos discutiendo. Los individuos responden a la interpelación porque reconocen (de donde parece salir la implicación de que "son ya sujetos") que es a ellos a quienes va dirigida. El mecanismo resulta así ignorado en la propia forma del reconocimiento, oculto detrás de la propia evidencia que lo mantiene en la existencia.

Tenemos así una primera parte de la respuesta a nuestro interrogante: los individuos se relacionan necesariamente de manera imaginaria con sus condiciones de existencia porque no hay otra opción para ellos que ser reclutados como sujetos y sólo aquellos con capacidad para encontrar una unidad imaginaria (evidencia y necesidad) donde no hay fundamentos para la misma son reclutados por la ideología, clasificados como sujetos. 
Hay, con todo, una razón de otro orden para comprender por qué esto ha de ser necesariamente así. Para ello tenemos que reparar en las consecuencias que posee el mecanismo de la interpelación y la correlativa constitución de los sujetos. Althusser establece este punto a través de la ambigüedad de la noción de sujeto, como sustantivo "sujeto (libre y responsable)" y como adjetivo "sujeto (sojuzgado)". En resumidas cuentas, al ser subjetivizados, los individuos son a su vez sojuzgados, fijados a un área de acción restringida:

\begin{abstract}
el individuo es interpelado como sujeto (libre) para que se someta libremente a las órdenes del Sujeto, por lo tanto, para que acepte (libremente) su sujeción, por lo tanto para que "cumpla sólo" los actos y gestos de su sujeción (...) es necesario que sea así, para que las cosas sean como deben ser, digámoslo ya, para que la reproducción de las relaciones de producción sea asegurada cada día (incluso en los procesos de producción y circulación) en la "conciencia", o sea, en el comportamiento de los individuos sujetos que ocupan los puestos que la división sociotécnica del trabajo les ha asignado en la producción, la explotación, la represión, la ideologización, la práctica científica, etc. (1970, p. 63).
\end{abstract}

Podemos ahora dar el paso final de esta parte de nuestra indagación. La ideología aparece como una categoría omnihistórica, en virtud de que la fijación de los individuos a esferas de actividad parece ser un requisito de toda sociedad. Este sujetamiento tiene que ocurrir, según Althusser, forzosamente a través de la categoría de sujeto, que involucra necesariamente la relación imaginaria de los individuos con los dispositivos que los configuran como sujetos. De aquí se desprende que tampoco hay otra opción para los ordenamientos sociales que reclutar a los individuos como sujetos. Por tanto, podemos comprender entonces que la relación de los individuos con sus condiciones de existencia deba ser pensada como necesariamente imaginaria.

\title{
2 LA FILOSOFÍA TERAPÉUTICA DE WITTGENSTEIN Y LA CONSTITUCIÓN DEL HABLANTE
}

Wittgenstein desarrolla en las Investigaciones filosóficas una propuesta filosófica bastante peculiar. Es que Wittgenstein no concibe a la filosofía como un cuerpo de doctrina, como una serie de tesis, hipótesis o leyes, sino como una forma 
de actividad. Esta forma de actividad es pensada por analogía con la labor terapéutica. La enfermedad con la que se confronta la terapia filosófica no es otra que la filosofía en sentido tradicional (la filosofía como cuerpo de doctrina). Sin embargo, Wittgenstein raramente se dirige a las concepciones particulares de uno u otro filósofo; en su lugar toma como su objeto de análisis lo que denomina figuras (Bilden, pictures), esto es, concepciones generales que reflejan nuestras actitudes fundamentales, en el sentido de que las mismas dan forma a nuestra inclinación a plantearnos ciertas preguntas y al tipo de respuestas que aceptaríamos como satisfactorias. Estas figuras son núcleos conceptuales rústicos, susceptibles de ser refinados de distintas maneras. ${ }^{11}$ Con todo, Wittgenstein confía en que cuando logramos percibir la inadecuación de una figura de esta clase, dejamos de estar dominados por la tentación de refinarla de una manera u otra. Una imagen o figura establece la manera en que las cosas "tienen que ser" ${ }^{\prime 2}$ grosso modo, de manera que mientras estamos cautivos de la misma sus fallos nos parecen cuestiones de detalle, subsanables con un refinamiento de nuestro conocimiento.

Al comienzo de las Investigaciones filosóficas Wittgenstein presenta una tal imagen del funcionamiento del lenguaje a partir de un fragmento de las Confesiones de Agustín, en el que éste recuerda su aprendizaje del lenguaje. El fragmento contiene una figura del funcionamiento del lenguaje y la comprensión. Wittgenstein lo comenta así:

\begin{abstract}
En estas palabras obtenemos, a mi parecer, una determinada figura de la esencia del lenguaje humano. Concretamente ésta: Las palabras del lenguaje nombran objetos -las oraciones son combinaciones de esas denominaciones- En esta figura del lenguaje encontramos las raíces de la idea: cada palabra tiene un significado. Este significado está coordinado con la palabra. Es el objeto por el que está la palabra. (IF § 1)
\end{abstract}

La comprensión del lenguaje consistiría, para Agustín, en colegir las conexiones entre palabras y cosas, de manera que una vez lograda la comprensión, la dificultad radicaría en adquirir la habilidad de proferir adecuadamente los signos para manejarse en el intercambio social. Como dice Agustín: "una vez adiestrada la lengua a esos signos [después de haber comprendido] expresaba ya con ellos mis deseos" (Confesiones, I, 8, cit. en Wittgenstein $1953 \S 1$ ).

\footnotetext{
11 La figura agustiniana puede reconocerse en concepciones sofisticadas, como las teorías semánticas de Frege o los atomismos lógicos de Russell y del Wittgenstein del Tractatus. Véase "Augustinian Picture of Language" en GLOCK, 1996, pp. 41-45.

12 "No digas: "Tiene que haber algo común a ellos o no los llamaríamos "juegos"” - sino mira si hay algo común a todos ellos" (WITTGENSTEIN, 1953, § 66).

Cad. de Pesq. Interdisc. em Ci-s. Hum-s., Florianópolis, v.12, n.101, p.4-30 ago/dez 2011
} 
Ahora bien, en la concepción agustiniana la comprensión y la acción (sea o no lingüística) aparecen como conceptualmente separables. Esto es, Agustín cree haber comprendido, en un primer momento, para sólo en un segundo momento, después de haber adiestrado su lengua a la pronunciación de los signos, realizar las acciones lingüísticas que se siguen de esa comprensión. Dicho de otra manera, el vínculo entre un término (la comprensión, la aprehensión de ideas) y el otro, la acción (en este caso lingüística), aparece como una relación externa, como un vínculo en el que ambos términos pueden concebirse uno con independencia del otro.

La respuesta wittgensteiniana a Agustín combina dos movimientos. Por un lado, diagnóstico y crítica; diagnóstico en el cual se intenta descubrir y describir cuáles son las bases a partir de las cuales se erige la figura del lenguaje que hemos descrito, que se combina con un movimiento crítico en el que se muestran las consecuencias absurdas a las que la misma conduce. Por otra parte un movimiento de reconstrucción o elucidación gramatical, en el que se intenta que quien está cautivo de una figura, "enfermo" de un problema filosófico, pueda reparar en aquello que, en cierto sentido, ya conoce, pero que, sin embargo, cuando intenta llevar a la representación eso conocido, revela de alguna manera no comprender. ${ }^{13}$

La concepción de la comprensión contenida en la observación de Agustín y que constituye el objetivo de la crítica wittgensteiniana en las secciones iniciales de las Investigaciones filosóficas ha recibido diferentes denominaciones. En este trabajo utilizaremos la denominación "paradigma mentalista tradicional". Bajo la misma pretendemos englobar los diferentes modos en los que la filosofía ha intentado encontrar tanto una ontología como una epistemología que haga justicia a algunas peculiaridades de nuestro comportamiento lingüístico. Hay un rasgo de nuestro comportamiento lingüístico que es especialmente relevante: usualmente no se nos presentan dudas acerca del sentido o el significado de las expresiones. Por ejemplo, no tenemos dudas acerca de lo que significa la expresión "Hay una montaña de oro en la costa uruguaya", mientras que la verdad de este enunciado es una cuestión legítimamente dudosa. En términos más clásicos, se trata de la distinción entre el carácter a priori de las cuestiones semánticas y el carácter a

\footnotetext{
${ }^{13}$ Wittgenstein señala: "Queremos entender algo que ya está patente ante nuestros ojos. Pues es esto lo que, en algún sentido, parecemos no entender." (1953, § 89).

Cad. de Pesq. Interdisc. em Ci-s. Hum-s., Florianópolis, v.12, n.101, p.4-30 ago/dez 2011
} 
posteriori de las cuestiones de hecho. Pues bien, ¿cómo se debe dar cuenta del carácter a priori de la comprensión, del conocimiento del significado? La vía más fatigada en términos filosóficos ha sido la de una ontología justificatoria. De acuerdo a la misma, habría un dominio, el del significado (ideas, conceptos, etc.) en relación al cual el sujeto poseería acceso privilegiado e inmediato, dotándolo de un conocimiento del que se desprenderían deductivamente los casos del significado en cuestión. La "concepción mentalista tradicional" es un ejemplo de esta estrategia.

Veamos ahora la crítica que Wittgenstein realiza a esta concepción, mostrando que la elucidación del conocimiento del significado en términos de una ontología justificatoria genera paradojas de una manera irremediable. La manera más sencilla de presentar la crítica de Wittgenstein a este modelo es a través de su análisis de las explicaciones o definiciones ostensivas. Una explicación ostensiva es la definición de un término que realizamos señalando un objeto y pronunciando al mismo tiempo la expresión que deseamos definir $(x)$ en cláusulas como "Esto es un $x$ ". En su análisis, Wittgenstein muestra que dichas definiciones no agotan las posibilidades de malentendidos. Por ejemplo, si señalo una hoja y digo 'hoja', mi alumno puede malentender mi explicación tomando a continuación objetos blancos de cualquier clase y profiriendo con muestras de satisfacción la expresión 'hoja'. Al respecto cabe decir dos cosas, en primer lugar, que esto es evidencia para decir que nuestro alumno nos interpretó mal (Wittgenstein indica: "cómo 'interpreta' ('auffasst') él la definición se muestra en el uso que hace de la palabra explicada" (1953 § 29)) y en segundo lugar, que si continuamos reflexionado sobre estos ejemplos, llegaremos a convencernos, con Wittgenstein, acerca de que "...la definición ostensiva puede en todo caso ser interpretada de manera diferentes" (1953 § 28).

Sin embargo, la crítica wittgensteiniana no termina aquí, sino que va un poco más profundo. En particular se dirige a la tendencia a considerar que cuando nuestro alumno comprendió erróneamente nuestra explicación, entonces, aparte de sus actos, ocurrió algo más, algo que tuvo lugar en su mente, en la cual interpretó 'hoja' como blanco, episodio mental del cual se deriva su acción. ${ }^{14}$ Wittgenstein explora también este terreno cuando de la indeterminación de las definiciones ostensivas

\footnotetext{
${ }^{14}$ Como dice Althusser: "la ideología de la ideología reconoce, a pesar de su deformación imaginaria, que las "ideas" de un sujeto humano existen o deben existir en sus actos, y si eso no sucede, le proporciona otras ideas correspondientes a los actos (aun perversos) que el sujeto realiza." (1970, p. 46; WITTGENSTEIN, $1953 \S 185$ ).

Cad. de Pesq. Interdisc. em Ci-s. Hum-s., Florianópolis, v.12, n.101, p.4-30 ago/dez 2011
} 
concluye que las mismas definen un término para quien ya conoce el papel de la palabra definida en el lenguaje (1953 § 30): alguien que sabe, por ejemplo, que la palabra definida es una palabra de color, pero desconoce a qué color específico hace referencia. Sólo que, una vez hecha esta salvedad, nos advierte Wittgenstein, surgen "todo tipo de cuestiones en relación con las palabras "saber" o "estar claro"” (1953 § 30). Lo que está en cuestión es el estatuto del conocimiento del significado (del conocimiento del uso del lenguaje, del conocimiento del papel de una palabra en el lenguaje): "Tiene uno que saber (o poder) algo [conocer el papel de una palabra en un juego de lenguaje, según dijimos antes] para poder preguntar por la denominación ¿pero qué tiene uno que saber?” (1953 § 30).

En consecuencia, Wittgenstein va a cuestionar la tentación nuclear de la figura agustiniana, que deriva la aplicación, los actos materiales, de ideas. Por un lado, va a mostrar que tales actos, lo que denominamos la "ontología subyacente a la comprensión", no son condiciones suficientes de la misma. Es decir, los presuntos actos de comprensión (la ontología subyacente) pueden estar presentes y sin embargo no haber comprensión. Por otra parte, Wittgenstein muestra que los actos de comprensión (en el sentido de la ontología mental subyacente) tampoco son condiciones necesarias, esto es, que la ontología subyacente puede estar ausente y haber sin embargo comprensión. El siguiente párrafo ilustra bien estos puntos:

\begin{abstract}
¿Qué es lo que realmente nos viene a la mente cuando entendemos una palabra? - ¿No es algo como una figura? ¿No puede ser una figura?

Bueno, supón que al oír la palabra "cubo" te viene a la mente una figura. El dibujo de un cubo, pongamos por caso. ¿Hasta qué punto esta figura puede ajustarse a un empleo de la palabra "cubo" o no ajustarse a él? Quizá digas: "eso es sencillo; - si me viene a la mente esa figura y señalo, por ejemplo, un prisma triangular y digo que es un cubo, entonces este empleo no se ajusta a la figura."- ¿Pero no se ajusta? He elegido el ejemplo intencionadamente de manera que sea muy fácil imaginarse un método de proyección según el cual la figura ajusta después de todo. (1953 § 139)
\end{abstract}

El ejemplo de este fragmento está construido de manera de socavar internamente al modelo mentalista tradicional. En primer lugar, el ejemplo presenta los aspectos cruciales de dicho modelo, esto es, en el mismo encontramos diferenciados con bastante claridad sus elementos. Por un lado, aparece la ontología de la comprensión ("la figura del cubo"), que cumpliría la función de distinguir un acto de comprensión de otro (si otra figura viniera a la mente se trataría de un acto de comprensión diferente). Por otra parte, la realización de juicios es entendida como 
algo que puede leerse inmediatamente en la comparación entre el significado y el caso al que el significado pretende aplicarse, en el cual la evidencia jugaría el papel de garantía racional (la aplicación sería así un ejercicio deductivo a partir del significado), representado en el ejemplo anterior como la "experiencia de ajuste". ${ }^{15}$

El ejemplo socava el modelo al presentar dos posibles aplicaciones de la figura del cubo que podríamos sentirnos igualmente inclinados a considerar como adecuadas. En el siguiente párrafo el propio Wittgenstein indica que lo que efectuó el ejemplo del $\S 139$ es recordarnos que: “... bajo ciertas circunstancias estaríamos dispuestos a llamar también "aplicación de la figura cúbica" a otro proceso, además de aquel en el que habíamos pensado originalmente." (1953 § 140). Esto es, dos aplicaciones diferentes, la aplicación a un objeto cúbico y la aplicación a un prisma triangular, podrían encajar con una y la misma experiencia (la contemplación mental de la figura de un cubo), identificada inicialmente como la experiencia de comprensión de 'cubo'.

Lo que hace inviable a la concepción mentalista tradicional es que es que la misma genera, de manera irremediable, una duplicidad de criterios potencialmente en contradicción entre sí. Este problema no es más que un reflejo de haber pensado desde el comienzo a la comprensión (estado mental) y a la aplicación (juicios) como términos independientes entre sí, como términos que guardan entre sí una relación externa. Sin embargo, el caso que consideramos permite extraer una lección: si el estado mental que identificamos en principio como la comprensión de 'cubo', (el "venirnos a la mente la figura del cubo") da lugar a una aplicación que contradice la identificación que habíamos hecho inicialmente (su aplicación a un prisma triangular, por ejemplo) ¿seguiremos diciendo que se trata del estado de comprender tal o cual cosa? La respuesta de Wittgenstein es clara, la aplicación es el criterio básico de la comprensión:

...lo esencial es que veamos que al oír la palabra puede que nos venga a la mente lo mismo y a pesar de todo ser distinta su aplicación. ¿Y tiene entonces el mismo significado las dos veces? Creo que lo negaríamos. $(1953 \S 140)$

\footnotetext{
${ }^{15}$ Wittgenstein analiza $(1958$, p. 122) cuál sería el elemento que representa a la evidencia en el modelo analizado, el que toma la forma de "una sensación específica de no ser capaz de distinguir" entre el ejemplo (el estado mental hipotéticamente identificado con el significar) y el material escogido, es decir, la aplicación, la circunstancia en la cual un juicio es realizado. La evidencia, concebida de esta manera, haría de la realización de juicios un ejercicio de razonamiento deductivo. Cad. de Pesq. Interdisc. em Ci-s. Hum-s., Florianópolis, v.12, n.101, p.4-30 ago/dez 2011
} 
La insistencia de Wittgenstein en que la aplicación es el criterio prioritario de la comprensión acarrea algo más, y es que atribuimos comprensión, es decir, comprensión correcta, a través de la concordancia en los juicios. En el $\S 29$ indica indirectamente que la falta de concordancia en los juicios es un criterio para denegar comprensión: "[Que una explicación adicional] sea necesaria en la definición ostensiva del dos depende de si sin esa palabra él la interpreta de modo distinto a como yo deseo." (1953) En el § 145 es más explícito aún: "Pero supongamos ahora que, tras algunos esfuerzos del maestro, él [el alumno] continúa la serie correctamente, es decir, como lo hacemos nosotros." (1953, énfasis añadido).

Las observaciones acerca del doble criterio implican un argumento antirreduccionista en el sentido de que cualquier propuesta reduccionista desconoce el carácter prioritario de la aplicación (por definición intenta buscar algo más básico que la misma) y por ello se ve enredada inevitablemente en el problema del doble criterio, que, según hemos argumentado, se resolverá siempre a favor de la aplicación.

Este abordaje abre una serie de problemas. Por un lado, la ruptura con la concepción de los significados como continentes de la aplicación y la necesidad de concordancia en los juicios parecen suprimir las relaciones internas que reconocemos existen entre la regla y sus casos, entre la comprensión y lo comprendido. La relación entre la serie de los pares y el paso 1002 como sucesor de 1000 no es una relación contingente, no es cuestión de esperar que los otros actúen para saber si este es el paso correcto. Wittgenstein es consciente de esta aparente consecuencia de su planteo, por ello sostiene: "A la comprensión por medio del lenguaje pertenece no sólo una concordancia en las definiciones, sino también (por extraño que esto pueda sonar) una concordancia en los juicios. Esto parece abolir la lógica; pero no lo hace." (1953 § 242)

Para encontrar una respuesta satisfactoria a este problema, es importante reparar en una característica aparentemente paradójica de la exposición de Wittgenstein. Nos referimos a que estos argumentos chocan una y otra vez con la incredulidad del propio Wittgenstein, en cuanto éste se dispone a hablar en primera persona. Esto hace que los mismos argumentos tengan que ser repetidos una y otra vez (a propósito de las definiciones ostensivas, a propósito de la comprensión de series matemáticas, acerca del lenguaje de las sensaciones, a propósito de la 
relación entre la voluntad y la acción, acerca de la visión de aspectos de una cosa, etc.) Vale la pena aclarar que la construcción argumentativa o retórica de las Investigaciones filosóficas ha sido caracterizada por K. T. Fann como una "confesión", en un sentido análogo a las Confesiones de Agustín, en la que predomina una dialéctica entre las 'voces de la tentación' y la 'voz de la corrección' (Véase FANN, 1997, pp. 127 y ss.; ver también CAVELL, 2002). La voz de la tentación aparece característicamente en primera persona del singular, como en este caso:

\begin{abstract}
“¿Pero cómo puede ser esto? [que la comprensión requiera de la concordancia en los juicios] ¡Cuando yo digo que entiendo la ley de una serie, seguro que no lo digo sobre la base de la experiencia de haber aplicado hasta ahora la fórmula algebraica de tal y cual manera! Seguro que sé en todo caso por mí mismo que significo la serie de este y aquel modo; da igual hasta dónde la haya desarrollado de hecho." - Quieres decir, pues: conoces la aplicación de la ley de la serie prescindiendo totalmente de un recuerdo de las aplicaciones efectivas a determinados números. $Y$ quizá digas: "iEvidentemente! Puesto que la serie es en efecto infinita y el trozo de serie que yo pude desarrollar, finito." (WITTGENSTEIN, $1953 \S$ 147).
\end{abstract}

Nótese que el intercambio entre las voces de la tentación y la de la corrección no conduce a una contradicción lisa y llana. Cuando "la voz de la tentación" reclama entender sin recurso al recuerdo de la aplicación concordante, e independientemente de cual haya sido la aplicación que de hecho ha realizado de la formula algebraica, "la voz de la corrección" no la contradice. No le dice, "pues te equivocas, el significado no es más que un constructo social, jamás sabrás cual es la respuesta correcta hasta ver el comportamiento de la comunidad en la que actúas" o cosa por el estilo. Hay una razón profunda para que las cosas sean de este modo en el intercambio: la voz de la tentación, más allá de su inadecuación como visión teórica, expresa uno de los requisitos constitutivos que hacen que alguien sea un ser hablante, uno de los requisitos sin los cuales eso que conocemos como lenguaje se vendría abajo. Dicho de otra manera, la dialéctica que estamos analizando debe mantenerse necesariamente en un delicado equilibrio: si la voz de la tentación fuera reemplazada por la voz de la corrección, el lenguaje se vendría abajo, ya que no se puede edificar un lenguaje sobre el conocimiento proposicional o teórico. La voz de la tentación, aunque equivocada desde el punto de vista teórico, no está entonces errada desde el punto de vista semántico ("Usar una palabra sin 
justificación (Rechtfertitung) no quiere decir usarla injustamente (Unrecht))" (WITTGENSTEIN, 1953, § 289).

Podemos clarificar este punto extremando las semejanzas con Althusser. Así, podemos decir que en el caso de Wittgenstein los individuos devienen hablantes "en virtud del funcionamiento de la categoría de significado". Por "funcionamiento de la categoría de significado" entendemos el ritual (el juego de lenguaje) de las afirmaciones semánticas. Las oraciones claves en este juego son oraciones categóricas como "Juan suma", "Yo sumo" y enunciados condicionales como por ejemplo: "Si Juan aprendió la suma, recuerda sus intenciones y desea acordarse a ellas, entonces, si se le presenta un problema como '57 + 68' responderá 125”.

El intérprete que a nuestro juicio mejor ha clarificado la lógica del ritual de las afirmaciones semánticas es Saul Kripke. De acuerdo a Kripke, la crítica de Wittgenstein al paradigma mentalista tradicional, y a otras propuestas de ontología subyacente afines al mismo, lleva a Wittgenstein a reemplazar la pregunta que dominaba este enfoque "¿Qué tiene que ocurrir para que las afirmaciones semánticas (como las citadas arriba) sean verdaderas?” por otras dos preguntas: “¿bajo qué circunstancias estas proposiciones pueden ser legítimamente afirmadas? y ¿cuál es la utilidad de dicha aseveración en nuestras vidas?” (KRIPKE, 2002, p. 73).

Veamos entonces qué pistas podemos recabar del estudio de las condiciones bajo las que afirmaciones semánticas pueden ser aseveradas legítimamente. Un enunciado categórico se atribuye, por ejemplo en una relación pedagógica, sobre la base de la concordancia de las respuestas del alumno con las que el maestro está dispuesto a dar. La atribución de un enunciado semántico categórico tiene entonces la forma de un "rito de pasaje", a través del cual alguien recibe un estatus social. Ahora bien, una de las condiciones a las que el maestro atenderá para atribuir el enunciado "Juan suma" es la seguridad práctica de Juan al continuar la serie, es decir, la ausencia de dudas acerca de cómo continuar. Por su parte, una vez recibido el estatus de sumante, de poseedor del concepto, tal es el caso del maestro o del alumno Juan. Una vez que ha superado exitosamente las pruebas que se le proponen y a las que se somete, el individuo puede dar por buenas, por correctas, 
las respuestas que se siente confiado a dar, sin más justificación que esa, estando en esto sin embargo sujeto a la corrección por otros. ${ }^{16}$

Veamos ahora cómo funcionan los enunciados semánticos condicionales. Allí donde la concepción mentalista tradicional señala que "porque comprendemos el mismo concepto actuamos del mismo modo", donde el énfasis está puesto en el antecedente del condicional (comprendemos), la elucidación terapéutica desplaza los énfasis, señalando la primacía de la práctica, haciendo lugar a la prioridad de la aplicación sobre la que hemos estado insistiendo. Esta prioridad se expresa, en el análisis terapéutico, elucidando el funcionamiento de los condicionales semánticos bajo la forma de un condicional contrapuesto, lo que en nuestro ejemplo sería: "si no responde 125 al problema 57+68, entonces no ha estado sumando", o en una versión un poco más refinada: "si no actuamos del mismo modo (de manera reiterada) entonces no (decimos que) comprendimos el mismo concepto". Lo que va entre paréntesis en esta fórmula intenta indicar que la misma no funciona de manera algorítmica, sino de manera aproximada (es a menudo suficiente que $X$ no actúe como lo esperamos para que digamos que $X$ no sigue la regla, etc.). Queda allí un espacio indeterminado, y por ello de disputa, que podemos pensar tal vez como uno de los terrenos de la lucha ideológica.

El ritual de los enunciados semánticos, el "funcionamiento de la categoría de significado", es entonces un ritual a través del cual otorgamos, conservamos y denegamos estatus sociales. El propósito de los mismos es clasificar a los individuos de acuerdo a las actividades que pueden o no realizar. La semejanza con la propuesta de Althusser es a esta altura evidente.

Queda entonces, para concluir esta sección, reparar en que lo que caracterizamos como "la voz de la tentación" se apoya sobre un rasgo necesario de nuestras prácticas lingüísticas: la seguridad práctica, la ausencia de dudas para hacer uso de una expresión simbólica, es un criterio del que nos valemos para hacer

\footnotetext{
${ }^{16}$ El siguiente párrafo introduce claramente la dualidad de perspectivas: "Para que pueda parecerme que la regla ha producido todas sus consecuencias por anticipado, éstas tienen que ser para mí obvias. Tan obvias como es para mí llamar "azul" a este color. (Criterios de que esto sea para mí 'obvio')." (WITTGENSTEIN, 1953, §238). Para quien comprende una regla las consecuencias se siguen naturalmente de la misma. A su vez, al juzgar si otro comprende la regla, se ha de tener el cuenta de si las consecuencia de la regla se siguen también para él naturalmente, si posee seguridad práctica al extraer las consecuencias de la regla, es decir, al realizar los juicios de los que decimos que son consecuencia de la regla, de manera que la ausencia de duda, la seguridad práctica, junto con el acierto en las respuestas constituyen los "criterios de que algo es obvio para alguien".

Cad. de Pesq. Interdisc. em Ci-s. Hum-s., Florianópolis, v.12, n.101, p.4-30 ago/dez 2011
} 
enunciados semánticos categóricos como "Él suma", "Él sabe (no sabe) que es un indicador de caminos"17, etc.

\section{CONCLUSIONES}

Para concluir deseamos sistematizar las analogías sobre las que hemos trabajado, para destacar finalmente una tensión que sordamente ha estado tensando a este trabajo.

Un buen punto para sistematizar las analogías entre ambos pensadores es lo que Althusser denomina "sistema cuádruple de la interpretación" en la estructura especular de la ideología" (1970, p. 62) mostrando análogos prima facie plausibles para cada uno de los cuatro momentos de esta estructura en términos de la filosofía wittgensteiniana. Para Althusser, la estructura especular de la ideología asegura simultáneamente:

1) la interpelación de los "individuos" como sujetos,

2) su sujeción al Sujeto,

3 ) el reconocimiento mutuo entre los sujetos y el Sujeto, y entre los sujetos mismos, y finalmente el reconocimiento del sujeto por él mismo.

4) la garantía absoluta de que todo está bien como está y de que, con la condición de que los sujetos reconozcan lo que son y se conduzcan en consecuencia, todo irá bien: "Así sea".(1970, p. 62)

Los dos primeros momentos de este sistema: (1) la interpelación de los individuos como sujetos y (2) la sujeción de los mismos al Sujeto, pueden ponerse en paralelo con el juego o ritual de las atribuciones semánticas, que tiene por resultado la sujeción del individuo al significado o a una regla, que hace del mismo un hablante integrado en un juego de lenguaje, es decir, en una comunidad de habla y actividades (juego de lenguaje; práctica). Por su parte, la relación de dependencia mutua entre el Sujeto y los sujetos, el punto (3) de Althusser, puede emparejarse

\footnotetext{
${ }^{17}$ Al fin del parágrafo acerca del indicador de caminos, Wittgenstein indica: "Así es que puedo decir que el indicador de caminos no deja después de todo ninguna duda abierta. O mejor: deja a veces una duda abierta y otras veces no. Y ésta ya no es una proposición filosófica, sino una proposición empírica." (1953 § 85) Que el indicador de caminos no deja ninguna duda abierta acerca de cómo debe ser seguido es una "proposición filosófica", es decir, expresa un criterio para afirmar si alguien comprende o no qué es un indicador de caminos. En otros términos: si el poste deja una duda abierta para alguien, entonces no es un indicador de caminos para esa persona. Naturalmente esto no afecta la cuestión empírica acerca de que habrá individuos que duden frente al indicador de caminos, es decir, que no comprendan su significado.
}

Cad. de Pesq. Interdisc. em Ci-s. Hum-s., Florianópolis, v.12, n.101, p.4-30 ago/dez 2011 
con la elucidación gramatical que exhibe una trama en la que los significados y las reglas no poseen una existencia sustantiva, pero en la cual la referencia a los mismos ("atribuciones semánticas" como "X sigue la regla tal o cual") tienen un rol constitutivo dual (de la regla y de los sujetos a la regla, a partir de la práctica como base de atribución). Y finalmente, el aspecto que Althusser denomina "garantía" (4), de acuerdo al cual en la mayoría de los casos los sujetos en el seno de los dispositivos ideológicos "marchan solos", remite a la "confianza" del individuo en el manejo de las expresiones simbólicas, la inclinación a continuar por su propia cuenta, un criterio para atribuir comprensión, según vimos en la elucidación gramatical wittgensteiniana.

Esta seguridad práctica y capacidad de continuar que acabamos de mencionar, son aspectos de la noción wittgensteiniana de "forma de vida". Esta noción tiene un carácter dual, en la medida en que podemos afirmar tanto que la forma de vida tiene por resultado que las consecuencias de una regla sean para los sujetos obvias, como que a través de la seguridad práctica que los individuos manifiestan en sus reacciones son integrados en una forma de vida. ${ }^{18}$

La alusión a la noción wittgensteiniana de "forma de vida" nos habilita para decir una palabra sobre "la paradoja althusseriana" a la que nos referimos antes. La noción de forma de vida, en cuanto conjunto de reacciones primitivas de las que depende la posibilidad del aprendizaje, nos abre la puerta para entender la adquisición del lenguaje por aquellos que no poseen el lenguaje. Considerada desde el punto de vista de la noción de forma de vida, la paradoja se nos revela como la

\footnotetext{
${ }^{18}$ El vínculo entre la seguridad práctica como criterio de la atribución de comprensión y la noción de forma de vida se deja percibir en el el siguiente comentario de la situación de aprendizaje: “¿Cómo aprende a entender este sistema [la serie de los números naturales en el sistema decimal]? - En primer lugar se le escriben series de números a modo de muestra y se le exhorta a copiarlas. (...) Y ya hay aquí una reacción normal y una anormal por parte del aprendiz.- Tal vez guiemos su mano primero al copiar la serie del 0 al 9; pero luego la posibilidad de comprensión dependerá de que continúe escribiendo independientemente." (WITTGENSTEIN 1953, § 143, énfasis añadido). Luego, al retomar este ejemplo Wittgenstein considera el caso quien, habiendo desarrollado la serie de los pares hasta 1000, reaccionara a la instrucción de continuar "de la misma manera" con 1004, 1008, 1012, etcétera. Al respecto sostiene: "Pudiéramos decir quizá en tal caso: Esta persona entiende por naturaleza esa orden, con nuestras explicaciones, como nosotros entenderíamos la orden: "Suma siempre 2 hasta 1000, 4 hasta 2000, 6 hasta 3000, etc.». Este caso sería semejante al de una persona que por naturaleza reaccionase a un gesto demostrativo de la mano mirando en la dirección que va de la punta del dedo a la muñeca en vez de en dirección a la punta del dedo." $(1953, \S 185$, énfasis añadido). Sin embargo, como los fragmentos analizados dejan en claro, tampoco la forma de vida cumple el rol de un fundamento del acuerdo en el significado, ya que la concordancia con nuestras reacciones es uno de los criterios a través de los cuales atribuimos o denegamos que alguien posee la misma forma de vida que nosotros.
}

Cad. de Pesq. Interdisc. em Ci-s. Hum-s., Florianópolis, v.12, n.101, p.4-30 ago/dez 2011 
explicación de los que son ya sujetos, es decir, la explicación dada desde el punto de vista de los sujetos, por tanto, la explicación que pertenece a la representación ideológica de la ideología. Sin embargo, desde el punto de vista provisto por la noción de forma de vida, por el contrario, obtenemos una respuesta muy diferente, que hace comprensible que cuando decimos que los individuos en cuestión son sujetos no es principalmente porque reconozcan que es a ellos a quienes la interpelación va dirigida, sino porque responden a la misma. Esta representación está, en nuestra opinión, fuera del orden de la representación ideológica de la ideología.

Hemos dado una versión wittgensteinianizada de Althusser y una visión althusserianizada de Wittgenstein. Ya es el momento de indicar que este intento está atravesado por una tensión que tuvimos que acallar para poder desarrollar la comparación. En determinado momento, al presentar el funcionamiento de la categoría de significado en la elucidación gramatical wittgensteiniana, nos vimos en la tentación de glosar la postura del vienés en los siguientes términos: "la concepción mentalista tradicional (la semántica filosófica) refleja la relación necesariamente imaginaria de los individuos con las condiciones sociales de su producción en tanto hablantes". Si no adoptamos esta formulación fue porque Wittgenstein insiste una y otra vez en que las concepciones filosóficas no son un asunto de la práctica, sino de la reflexión sobre la práctica. Así sostiene Wittgenstein:

\footnotetext{
Prestamos atención a nuestros propios modos de expresión concernientes a estas cosas, pero no los entendemos, sino que los malinterpretamos. Somos, cuando filosofamos, como salvajes, hombres primitivos, que oyen los modos de expresión de hombres civilizados, los mal interpretan y luego extraen las más extrañas conclusiones de su interpretación. (1953 § 194)
}

Es cierto que Wittgenstein entiende que las causas de la confusión filosófica son en última instancia inducidas por nuestras formas de expresión, por las analogías superficiales entre las mismas, y que lo que nos lleva a enredarnos en tales confusiones no es algo diferente que aquello que nos permite capacitarnos como hablantes (forma de vida, inclinación a continuar confiadamente, etc.). Pero no considera que las mismas sean algo necesario. La expresión "Ahora sé como continuar" proferida en el curso del aprendizaje de una expresión simbólica, una serie matemática por ejemplo, y aún cuando gran parte del esfuerzo de Wittgenstein 
se dirige a mostrar que no hay fundamentos posibles (en el sentido de condiciones necesarias y suficientes) para conocer la continuación de una serie matemática, no es a su juicio algo necesariamente ilusorio. Esta expresión es una exclamación, cuyo dominio se adquiere de manera semejante a cómo se adquiere el dominio de otras exclamaciones, de dolor y placer por ejemplo, cuya adquisición se acredita socialmente, sujeta a la corrección por otros (ver Wittgenstein 1953, § 244). El dominio de tales expresiones no implica esencialmente ilusión, aunque constituye una piedra con la que alguien difícilmente deje de tropezar al reflexionar sobre el lenguaje, ya que por su forma parecen un caso de saber, utilizan la misma expresión que en los casos en que conocemos cuestiones de hecho ("Ahora sé como continuar" es análogo en su forma -en su gramática superficial- a "Ahora sé que vestido lleva puesto", aunque radicalmente distinto en su funcionamiento -en su gramática profunda-), parece excluir lógicamente la duda (la expresión "sé como desarrollar la serie matemática pero no sé cual es el próximo caso" carece de sentido), toda una serie de factores que generan la impresión de estar aludiendo a una certeza de orden superior (el carácter a priori del conocimiento del significado).

La elucidación terapéutica intenta entonces que podamos reconocer, separando lo que es dato de lo que es interpretación, la piedra sobre la que se levantan las concepciones filosóficas. La ausencia de duda en el manejo de las expresiones simbólicas es un rasgo constitutivo del ser hablante, un rasgo necesario, pero no porque esté fundada en un dominio de acceso privilegiado del que habríamos de concluir que los hablantes necesariamente poseen, como si alguien dijera: "sin ese saber no se puede hablar", sino porque constituye uno de los criterios con los son seleccionados los hablantes, uno de los criterios que está integrado en los rituales a través de los que funciona la "categoría del significado". ${ }^{19}$ El dominio de acceso privilegiado que sustentaría un conocimiento especial que se manifestaría en la certeza en relación al significado, es una proyección del dato de la ausencia de duda que el ritual de la categoría de significado utiliza para seleccionar a los hablantes.

La tensión que señalamos acaso sea la misma que se puede apreciar entre la brillante fórmula de Althusser, quien sostiene que: “...la ideología no tiene afuera

\footnotetext{
${ }^{19}$ En mi trabajo muestro la manera en que este dato es comprendido en el Tractatus y cómo las Investigaciones filosóficas constituyen, en este sentido, una completa inversión de aquella obra.

Cad. de Pesq. Interdisc. em Ci-s. Hum-s., Florianópolis, v.12, n.101, p.4-30 ago/dez 2011
} 
(para ella), pero al mismo tiempo, que no es más que afuera (para la ciencia y la realidad)" (1970, p. 56) y la parcialmente concordante formula de Wittgenstein, quien sostiene por su parte: "La filosofía expone meramente todo y no explica ni deduce nada.- Puesto que todo yace abiertamente, no hay nada que explicar. Pues lo que acaso esté oculto, no nos interesa." (1953, § 126). 


\section{REFERENCIAS}

ALTHUSSER, L. Ideología y aparatos ideológicos de estado. Freud y Lacan.

Trad. de Alberto J. Pla y José Sazbón. Buenos Aires: Nueva visión, 1988.

BALIBAR, E. El concepto de corte epistemológico de Gaston Bachelard a Louis Althusser. En: Escritos por Althusser. Trad. de H. Cardoso. Buenos Aires: Nueva Visión, 2004a.

BALIBAR, E. El no contemporáneo. En: Escritos por Althusser. Trad. de $\mathrm{H}$. Cardoso. Buenos Aires: Nueva visión, 2004b, pp. 86-87.

CAVELL, S. The Availability of Wittgenstein's Later Philosophy. En: CAVELL, S. Must We Mean What We Say. Cambridge: Cambridge University Press, 2002.

FANN, K. T. El concepto de filosofía en Wittgenstein. Madrid: Tecnos, 1997.

GLOCK, H-J. Wittgenstein Dictionary. Oxford: Blackwell, 1996.

KARSZ, S. Lectura de Althusser. Buenos Aires: Galerna, 1970.

KRIPKE, S. Wittgenstein on Rules and Private Language, Cambridge (Mass.): Harvard Univ. Press, 2002.

LACAN, J. El estadio del espejo como formador de la función del yo (je) tal como se nos revela en la experiencia psicoanalítica. En: ŽlŽEK, S. (ed.). Ideología. Un mapa de la cuestión. Buenos Aires: FCE, 2005.

WITTGENSTEIN, L. Investigaciones filosóficas. Trad. de U. Moulines y A. García Suárez. Barcelona: Altaya, 1999.

WITTGENSTEIN, L. Cuadernos azul y marrón. Trad. de F. García Guillén. Barcelona: Planeta-Agostini, 1994.

Artigo:

Recebido em: 06/09/2011

Aceito em: 08/11/2011 